\title{
Small intestinal vascular malformation bleeding: diagnosis by double-balloon enteroscopy combined with abdominal contrast-enhanced CT examination
}

\author{
Jun Cui, Liu Ye Huang, Cheng Rong Wu \\ Department of Gastroenterology, Yan Tai Yu Huang Ding Hospital, Yantai 264000, Shandong, China
}

\begin{abstract}
Aim: The purpose of this study was to explore the value of double-balloon enteroscopy combined with abdominal vascular-enhanced CT examination for the diagnosis of intestinal vascular malformation bleeding, to explore a simple and effective method for the diagnosis of small intestinal vascular malformation bleeding.

Methods: Ten patients with intestinal bleeding were first examined with double-balloon enteroscopy. If active bleeding considered as vascular malformation was observed, the patient underwent abdominal vascularenhanced CT examination. If no active bleeding was observed with double-balloon enteroscopy, the patient also underwent abdominal vascular-enhanced CT examination. When intestinal vascular malformation bleeding was diagnosed with double-balloon enteroscopy and/or abdominal vascular-enhanced CT examination, the patient underwent surgical operation and vascular malformation was confirmed with pathologic diagnosis.

Results: In ten patients who underwent double-balloon enteroscopy examination, active intestinal bleeding was observed in seven patients and no active bleeding was observed in three patients. All ten patients underwent abdominal vascular-enhanced CT examination and vascular malformation was detected in all the patients with confirmation by pathologic diagnosis.

Conclusion: Double-balloon enteroscopy combined with abdominal vascular enhanced CT examination is a simple and effective method for the diagnosis of intestinal vascular malformation bleeding.
\end{abstract}

Correspondence to: Jun Cui; email: cuijun89@163.com
Key words: Small intestine-Vascular malformation-Double-balloon enteroscopyMulti-slice spiral CT_-Diagnosis

Small intestinal bleeding is often the first symptom of small intestinal disease. Since the small intestine is about 5-6 m long, occupying a large zone in the abdominal cavity, conventional endoscopy and X-ray studies cannot make a thorough examination [1-3]. Double-balloon enteroscopy may provide adequate examination of the small intestine [4-6]. However, there are some patients whose small intestinal bleeding lesion may not be detected with enteroscopy. Since there is a "blind region" to both sides, especially when for the bleeding is caused by a vascular malformation; it then generally depends on angiography, but this has the limitations of an invasive procedure time consumption and high cost [7-9]. Recently, we used the method of contrast-enhanced CT combined with enteroscopy for the diagnosis of small intestinal vascular malformation in ten patients. Our experience is reported as follows.

\section{Materials and methods}

\section{General information}

Ten patients were diagnosed as gastrointestinal bleeding in our department from March 2006 to October 2010. No bleeding lesions were detected with gastroscopy and colonoscopy. They underwent enteroscopy through mouth and/or anus. In the patients with active bleeding lesions suspected of small intestinal vascular bleeding, abdominal vascular-enhanced CT examination was performed. The patients without active bleeding lesions also underwent abdominal contrast-enhanced CT examination. Among the ten patients, there were seven males and 
three females, age range was 35-65 years, mean age was 51 years. All the patients lacked the contraindications for enteroscopy, including severe heart, lung, and liver disease, severe weakness, and complete intestinal obstruction.

\section{Instruments}

Double-balloon enteroscopy with the Fujinon EN450P5/20 (Fujinon company, Japan) was accomplished with the repeated charge and discharge of the balloons, and the repeated push and pull of the trocar sheath to advanced the enteroscope [10].

\section{Preparation before examination}

Electrocardiogram, blood tests, liver and renal function, blood type, and blood coagulation tests were performed for the patients. Twelve hours of fasting was necessary for examination through mouth, small intestine clearing was necessary for examination through anus. Liquid diet the day before examination, 33\% magnesium sulfate of $100 \mathrm{~mL}$ at $8 \mathrm{pm}$ the night before examination, followed 30 min later by drinking $2000 \mathrm{~mL}$ of water. Disoprofol was injected $10 \mathrm{~min}$ before examination for the patients who needed intravenous anesthesia. For the patients who needed no intravenous anesthesia, ansiolin of $10 \mathrm{mg}$, scopolamine butylbromide of $10 \mathrm{mg}$, and dolantin of $50 \mathrm{mg}$ were injected $10 \mathrm{~min}$ before examination. Oxygen supply, electrocardiogram, and blood oxygen saturation monitoring were performed through the whole procedure.

\section{Method for enteroscopy}

The assistant and doctor cooperate to fulfill the procedure: in enteroscopy examination through the mouth the enteroscope progresses to the inferior segment of the ileum: the enteroscope advances to the junctional zone of the jejunoileum. When a bleeding lesion was not detected through one side, diluent methylene blue of $0.5 \mathrm{~mL}$ was injected beneath the mucous membrane at several sites, which facilitates confirming the site when the examination is performed through the other side. For some patients, X-ray was used to observe the extent of penetration by the enteroscope $[11,12]$.

\section{Abdominal contrast-enhanced CT examination}

Light speed VCT 64 layer spiral CT (GE company, USA) was used, scan range included the entire abdomen, plain scan was performed first, parameters: tube tension was $120 \mathrm{kV}$, tube current was $36 \mathrm{mAs}$, width of collimator was $40 \mathrm{~mm}$, rack rotation time was $0.6 \mathrm{~s} /$ circle, pitch was $1.375: 1$, and slice thickness was $1.25 \mathrm{~mm}$. Then enhancement scanning was performed, non-ionotropic contrast agent of ultravist 370 was used, injected as a bolus through a peripheral vein, the dosage was $1.5 \mathrm{~mL} /$ $\mathrm{kg}$ body weight, the flow rate was $3.5-4.5 \mathrm{~mL} / \mathrm{s}$. After enhancement scanning, image reconstruction was performed with the original data, after reconstruction all the images were transmitted to the work station, where post processing was taken, including multi-planar reformation (MPR) and curved planar reconstruction (CPR). The best images were screened for analysis and diagnosis.

\section{Analysis of CT images}

The CT images were separately analyzed by two radiologists with rich experience; when the conclusion was not the same, the two radiologists discussed the images until the same conclusion was reached. The images of enteroscopy and CT were compared with the surgical result. The clinical value of applying multi-slice spiral CT (MSCT) in the diagnosis of small intestinal vascular malformation was evaluated.

\section{Results}

Small intestinal bleeding detected with doubleballoon enteroscopy

Of the ten patients who underwent double-balloon enteroscopy examination, active bleeding lesions were detected in seven patients, and no active bleeding sites were detected in three patients (shown in Fig. 1, Table 1).

\section{Small intestinal vascular malformation detected with abdominal contrast-enhanced $C T$}

Of the ten patients who underwent contrast-enhanced CT, small intestinal vascular malformations were detected in all and these were confirmed with surgical and pathologic results (shown in Table 1).

\section{Surgical detection and treatment measure}

All the ten patients with intestinal vascular malformation underwent surgical operation. The vascular malformation was confirmed with surgical detection and pathologic diagnosis, and segmental resection was done for intestinal vascular malformation and angioma (shown in Table 1).

\section{Typical case}

Patient no. 2 had undergone a surgical operation 4 years previously for a cutaneous hemangioma of the right knee (Fig. 2A). He was admitted for dark stools. Several jejunal angiomas were detected with enteroscopy through the mouth (Fig. 2B), but no active bleeding was observed. No lesion was detected with enteroscopy through the anus. Ileal vascular malformation was 


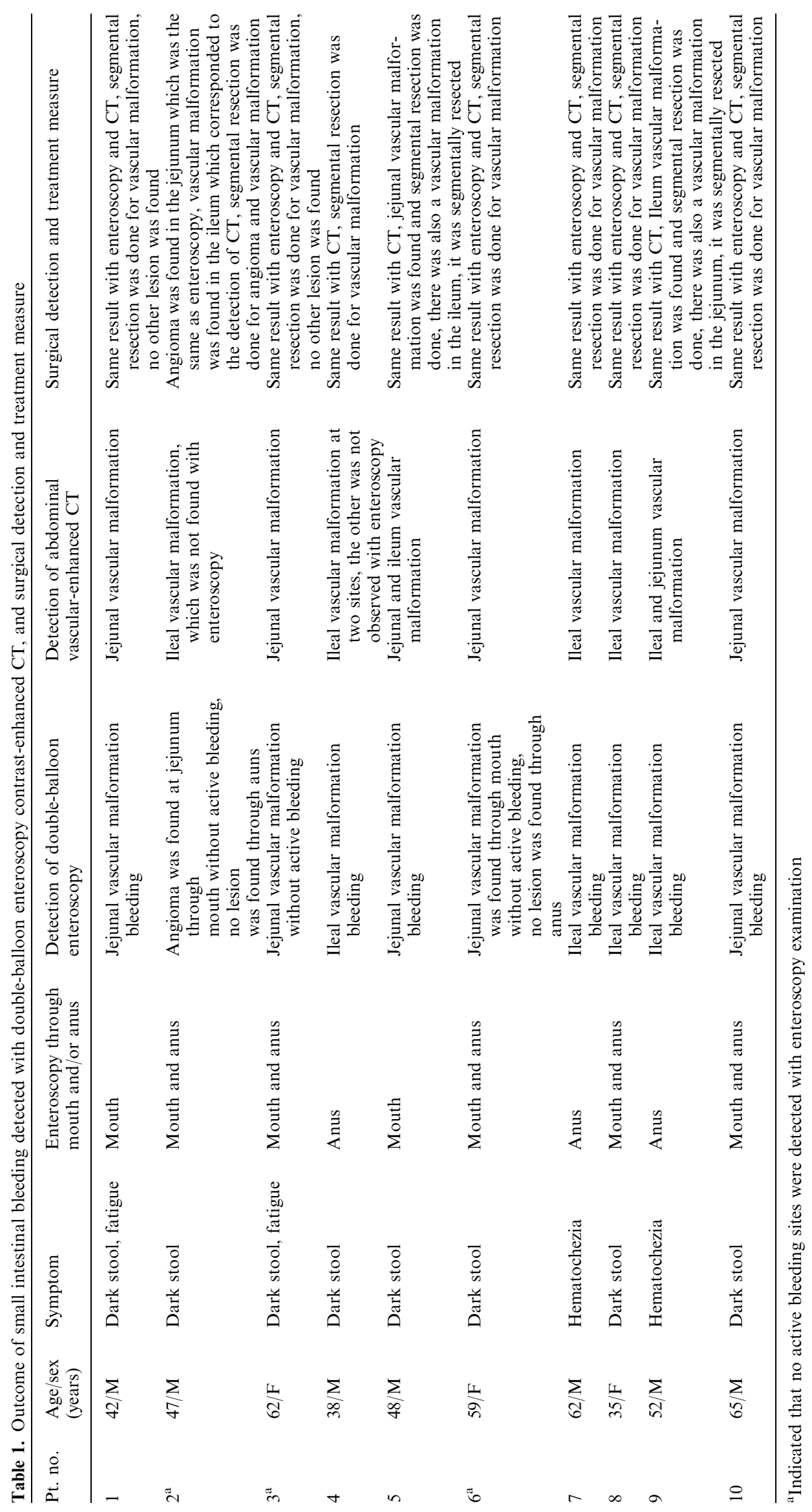



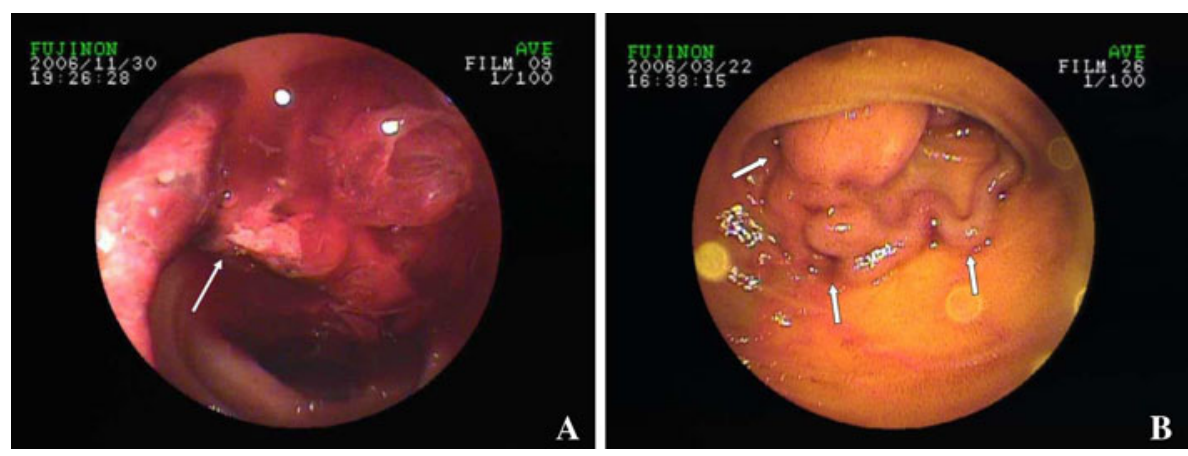

Fig. 1. Small intestinal vascular malformation detected by doubleballoon enteroscopy. A Jejunal active bleeding caused by arteriovenous malformation (patient no. 1). B Jejunum vascular ectasia, no active bleeding was observed, distorted blood vessel was detected (patient no. 3).

detected with abdominal contrast-enhanced CT (Fig. 2C-E). Ileal angioma (Fig. 2F) and vascular malformation (Fig. 2G) were detected with laparoscopic approach. Segmental resection was done for the angioma and vascular malformation, and the lesions were confirmed with pathologic diagnosis (Fig. 2H).

\section{Discussion}

Small intestinal bleeding refers to a bleeding lesion from the Treitz ligament to the ileocecal junction, generally due to peptic duodenal erosion or ulcer, diverticulosis of the small intestine, small intestinal vascular malformation or tumor, or inflammatory bowel disease. Because ordinary methods have limitations for small intestinal examination, the diagnosis of small intestinal bleeding is difficult [13-15].

Barium meal examination is useless for the diagnosis of mucous membrane and vascular disease of the small intestine. Radioisotope scanning is useful for the diagnosis of gastrointestinal bleeding, the tracer commonly used is 99Tcm and 99Tcm RBC. 99Tcm can detect lesion bleeding at the speed of $0.05 \mathrm{~mL} / \mathrm{min}$, but since its half life is only about $3 \mathrm{~min}$, it misses intermittent bleeding. Moreover, the high radioactivity of liver and spleen may interfere with the diagnosis at these sites, so its sensitivity is decreased. 99Tcm RBC has some value for the diagnosis of intermittent bleeding, but the blood pool background at sites of abdominal vessels, liver, spleen, and soft tissue is relatively high; delayed imagings at $3 \mathrm{~h}, 6 \mathrm{~h}$, even $24 \mathrm{~h}$ are necessary [16].

Selective angiography is useful for the diagnosis of vascular malformation of the small intestine. Extravasation of contrast medium indicates that the bleeding speed is at least $0.5-1.0 \mathrm{~mL} / \mathrm{min}$. Especially useful when there is active bleeding, the positive rate is about 50\%-77\% [7].

When the lesion causing obscure lower digestive tract bleeding by intestinal disease is not diagnosed, it cannot not be properly treated, and exploratory laparotomy may become necessary. Detection of the lesion may remain unclear and the re-bleeding rate of post-operation is high. In the recent years, small intestinal bleeding was diagnosed with enteroscopy examination, because enteroscopy examination had little trauma, it was widely used in clinic. However, not all the lesions of the patients with small intestinal bleeding can be diagnosed with double-balloon enteroscopy $[17,18]$; even if enteroscopy through both mouth and anus is undertaken, there remains a "blind region". The diagnosis for the "blind region" bleeding mainly depends on angiography. With the advent of spiral CT, abdominal vessels can be clearly seen with enhanced CT after image reconstruction, replacing angiography to some extent, meanwhile, the procedure was brief, less invasive, and less demanding [19-22]. In this study, five patients underwent enteroscopy through both mouth and anus, and the enteroscopy and CT result was not the same in one patient (patient no. 2). In clinic, when the patients were diagnosed as intestinal vascular malformation bleeding through mouth or anus, a number of them would not accept enteroscopy examination through the other side for economic or other reasons, and they underwent surgical operation for bleeding. If contrast-enhanced CT was not done, some intestinal vascular malformations could be missed. In this study, for patient nos. 2, 4, 5, and 9, vascular malformation was diagnosed with contrast-enhanced CT, while the malformation was not observed with enteroscopy examination. Thus, we recommend that for the patients with small intestinal bleeding, whether or not bleeding sites were detected with enteroscopy, abdominal contrast-enhanced CT should be done before surgical operation to avoid the miss of other vascular malformations. Not all instances of small intestinal bleeding can be detected with enhanced CT [23]. In our department, some patients suspected as small intestinal bleeding underwent enteroscopy and enhanced CT examination, but the bleeding site was not detected; the reason perhaps was that the bleeding site was too small to be detected with enhanced CT. In our experience, abdominal-enhanced CT was accurate for the diagnosis of vascular malformation, but sometimes it could not differentiate small intestinal stromal tumor. Thus, enteroscopy and enhanced CT should be combined for the diagnosis of intestinal vascular malformation bleeding.

In recent years, we have used contrast-enhanced CT for the diagnosis of small intestinal vascular malforma- 

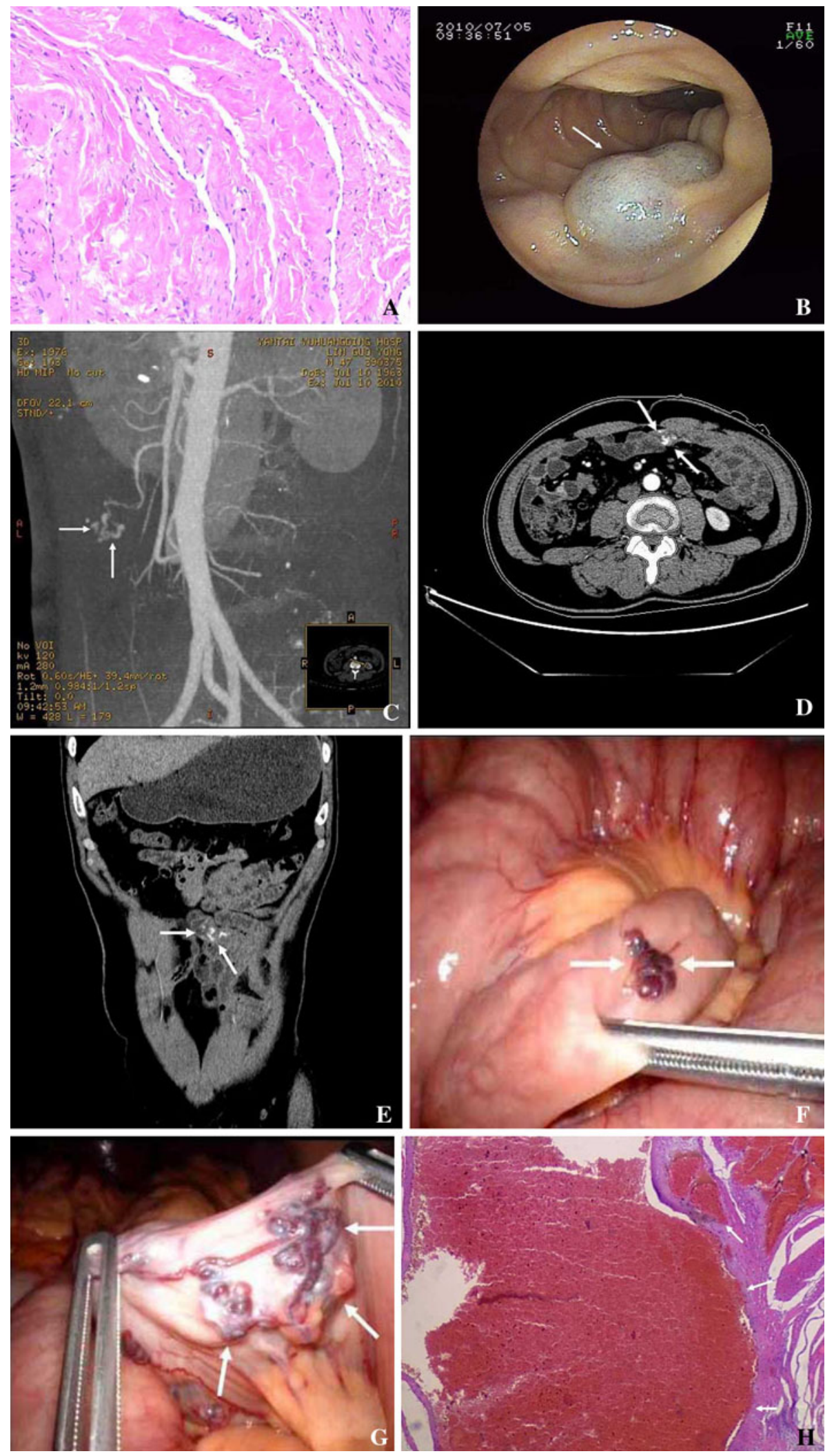

Fig. 2. Skin, enteroscopy, abdominal contrast-enhanced CT, laparoscopic, and pathologic findings. A Histology shows cavernous angioma of the right knee $(\times 400)$. B Angioma shown by enteroscopy examination through the mouth. C Arterio-venous malformation at a branch of the superior mesenteric artery shown by contrast-enhanced CT. D Contrast medium stays in the arterio-venous malformation on axial CT. E Contrast medium stays in the arterio-venous malformation shown by CT on coronal view reconstruction. $\mathbf{F}$ Small intestinal angioma (arrows) shown by laparoscopy corresponding to $\mathbf{B}$. G Arterio-venous malformation (arrows) shown by laparoscopic approach, corresponding to C-E. H Histologic section of resected small intestinal arterio-venous malformation: vessel wall is irregular, distorted and dilated $(\times 400)$. tion bleeding. With image reconstruction, small intestinal vascular malformation can be clearly seen and properly diagnosed. All the vascular malformations in this experience were confirmed with surgical operation and pathologic diagnosis. It was proved that abdominal contrast-enhanced CT has practical value for the 
diagnosis of small intestinal vascular malformation, making up the shortcomings of double-balloon enteroscopy.

Open Access. This article is distributed under the terms of the Creative Commons Attribution Noncommercial License which permits any noncommercial use, distribution, and reproduction in any medium, provided the original author(s) and source are credited.

\section{References}

1. Trifan A, Singeap AM, Cojocariu C, et al. (2010) Small bowel tumors in patients undergoing capsule endoscopy: a single center experience. J Gastrointestin Liver Dis 19:21-25

2. Gerson LB (2009) Outcomes associated with deep enteroscopy. Gastrointest Endosc Clin N Am 19:481-496

3. Pennazio M (2009) Enteroscopy in the diagnosis and management of obscure gastrointestinal bleeding. Gastrointest Endosc Clin N Am 19:409-426

4. Voelkel JP, Di Palma JA (2010) Deep enteroscopy. South Med J 103:1045-1048

5. Thomson M, Venkatesh K, Elmalik K, et al. (2010) Double balloon enteroscopy in children: diagnosis, treatment, and safety. World $\mathbf{J}$ Gastroenterol 16:56-62

6. Ramchandani M, Reddy DN, Gupta R, et al. (2009) Diagnostic yield and therapeutic impact of single-balloon enteroscopy: series of 106 cases. J Gastroenterol Hepatol 24:1631-1638

7. Hansel SL, Decker GA, Shiff AD (2009) Thirty years of overt, obscure GI bleeding solved by modern technology. Gastrointest Endose 70:595-597

8. Maganty K, Farrell RL (2009) Electronic clinical challenges and images in GI. Diagnosis: gastroduodenal artery pseudoaneurysm complicating as obscure gastrointestinal bleeding and acute liver failure. Gastroenterology 136:e1-e2

9. Gerson L, Kamal A (2008) Cost-effectiveness analysis of management strategies for obscure GI bleeding. Gastrointest Endosc 68:920-936

10. Mönkemüller K, Neumann H, Meyer F, et al. (2009) A retrospective analysis of emergency double-balloon enteroscopy for small-bowel bleeding. Endoscopy 41:715-717

11. John S, Appleyard M (2008) Role of double balloon enteroscopy in obscure gastrointestinal bleeding. J Gastroenterol Hepatol 23:994 996
12. Bellutti M, Fry LC, Schmitt J, et al. (2009) Detection of neuroendocrine tumors of the small bowel by double balloon enteroscopy. Dig Dis Sci $54: 1050-1058$

13. Kameda N, Higuchi K, Shiba M, et al. (2008) A prospective, singleblind trial comparing wireless capsule endoscopy and double-balloon enteroscopy in patients with obscure gastrointestinal bleeding. J Gastroenterol 43:434-440

14. Li X, Dai J, Lu H, et al. (2010) A prospective study on evaluating the diagnostic yield of video capsule endoscopy followed by directed double-balloon enteroscopy in patients with obscure gastrointestinal bleeding. Dig Dis Sci 55:1704-1710

15. Pasha SF, Leighton JA, Das A, et al. (2008) Double-balloon enteroscopy and capsule endoscopy have comparable diagnostic yield in small-bowel disease: a meta-analysis. Clin Gastroenterol Hepatol 6:671-676

16. Dolezal J, Vizda J (2008) Experiences with detection of the ectopic gastric mucosa by means of Tc-99m pertechnetate disodium scintigraphy in children with lower gastrointestinal bleeding. Eur J Pediatr Surg 18:258-260

17. Cellier C (2008) Obscure gastrointestinal bleeding: role of videocapsule and double-balloon enteroscopy. Best Pract Res Clin Gastroenterol 22:329-340

18. Hsu PS, Chen JL, Yu JC, et al. (2008) Accurate diagnosis and successful treatment for massive obscure small intestinal bleeding by means of intra-operative enteroscopy: a case report. Eur $\mathbf{J}$ Gastroenterol Hepatol 20:139-141

19. Yamaguchi T, Yoshikawa K (2003) Enhanced CT for initial localization of active lower gastrointestinal bleeding. Abdom Imaging 28:634-636

20. Yoon W, Jeong YY, Kim JK (2006) Acute gastrointestinal bleeding: contrast-enhanced MDCT. Abdom Imaging 31:1-8

21. Jaeckle T, Stuber G, Hoffmann MH, et al. (2008) Acute gastrointestinal bleeding: value of MDCT. Abdom Imaging 33:285-293

22. Duchat F, Soyer P, Boudiaf M, et al. (2010) Multi-detector row CT of patients with acute intestinal bleeding: a new perspective using multiplanar and MIP reformations from submillimeter isotropic voxels. Abdom Imaging 356:296-305

23. Stuber T, Hoffmann MH, Stuber G, et al. (2009) Pitfalls in detection of acute gastrointestinal bleeding with multi-detector row helical CT. Abdom Imaging 34:476-482 